\title{
Redundant Wavelet Watermarking using Spread Spectrum Modulation
}

\author{
V. H. Mankar, T. S. Das, S. Sarkar and S. K. Sarkar \\ Department of Electronics and Telecommunication Engineering, Jadavpur University, Kolkata INDIA \\ Received $09^{\text {th }}$ October 2007; revised $18^{\text {th }}$ March 2008; accepted $25^{\text {th }}$ March 2008
}

\begin{abstract}
Spread Spectrum modulation has become a preferred paradigm in many watermarking applications. This paper analyzes the performance of such a blind watermarking scheme under discrete wavelet frame rather than a traditional orthonormal wavelet expansion. The over complete representation offered by the redundant frame facilitates the identification of significant image features via a simple correlation operation across scales. The performance and resiliency of the proposed technique are analyzed against several volumetric distortion sources. The experimental results of this oblivious algorithm illustrate better visual and statistical imperceptibility and robustness compared to the usually critically sampled discrete wavelet transform. This algorithmic architecture utilizes the existing allocated bandwidth in the data transmission channel in a more efficient manner.
\end{abstract}

Key Words: Spread Spectrum Modulation, Discrete Wavelet Frame (DWF), Over Complete Representation, Redundant Wavelet Transform (RDWT), Image Watermarking.

\section{Introduction}

Image Watermarking is becoming increasingly of interest in tasks such as copyright control, image identification, fingerprinting, covert communication, broadcast monitoring, content verification and data hiding. Due to the explosion in the use of digital media, watermarking has recently drawn significant attention from academia and industry alike [1]. A number of transform domain techniques have been implemented for robust image watermarking. Spread Spectrum (SS) watermarking, one of the most popular and widely employed method embeds a watermark sequence into coefficients of an orthonormal or biorthogonal transform. The appealing DWT is an effective venue for SS watermarking due to the natural similarities between space-frequency tiling of the DWT and the operating characteristics of the human visual system (HVS) [8-12]. But this DWT decomposition can not efficiently characterize all the directions due to its separable nature. Another drawback is that DWT is not invariant to the integer shift. Moreover, DWT requires such decomposition filters transfer functions, which do not overlap with each other and are confined within rectangular regions in the frequency domain. All these aspects of DWT are connected in conflicting manner and leads to the use of non-orthogonal basis set. Here, alternative wavelet transform paradigms exist and can meet all the above cited requirements. In essence, RDWT, often implemented as the algorithme $\grave{a}$ trous removes the down sampling operation from the DWT to produce an over complete representation of the input sequence which functions to a certain extent as an approximation to the continuous wavelet transform. From a mathematical perspective, the RDWT is shift invariant and its redundancy introduces an

Correspondence to: <sksarkar@etce.jdvu.ac.in>

Recommended for acceptance by João Manuel R. S. Tavares and Renato Natal Jorge ELCVIA ISSN: 1577-5097

Published by Computer Vision Center / Universitat Autonoma de Barcelona, Barcelona, Spain 
over complete frame expansion have long been known to be robust to additive noise. Specifically, noise added in transform domain results in significantly reduced noise power in original signal domain due to the fact that the inverse frame operator is a pseudo-inverse that involves a projection onto the range space of the forward transform. Intuitively, RDWT based signal processing would be ideally suited to SS watermarking procedure. Indeed more watermarking energy can be accommodated in RDWT domain for the same distortion incurred in the original signal domain as compared to the DWT based watermarking [2]. In this paper, we conduct an investigation on RDWT that is essentially an undecimated DWT in the context of designing a robust SS image-watermarking scheme. In image coding area, we utilize its shift invariant property, which is not provided by dyadic wavelet transform, to implement high performance image coding system. While in image adaptive SS watermarking area, the RDWT provides more accurate estimation on locations where watermarks can be properly embedded. Thus, using RDWT, we can hide more watermarks than its traditional, critically sampled counterpart approach without harming the non-perceivable requirements. Specifically in the proposed method, the redundancy inherent in the transform facilitates the detection of perceptually salient features and guides the placement of watermarking energy so as to minimize the perceptual image quality.

The paper is organized as follows: Section 2 introduces the Redundant Discrete Wavelet Transform. The proposed watermarking architecture and its implementation are presented in the section 3 . In section 4 , we investigate the simulation results on $16 \times 16$ binary watermark and analysis of the obtained results. Finally, section 5 concludes and remarks about some of the aspects analysed in the present work.

\section{Redundant Discrete Wavelet Transform}

The RDWT has a extensive history, having been independently revealed a number of times and given a number of different names, including the algorithme à trous, the undecimated DWT (UDWT), the over complete DWT (ODWT), the shift-invariant DWT (SIDWT), and discrete wavelet frames (DWF). There are several ways to implement the RDWT, and several ways to represent the resulting over complete set of coefficients. The original implementation was in form of the algorithme à trous, which, in essence, removes the down sampling operator from the usual implementation of the DWT [3, 10]. The RDWT, which is employed in our anticipated watermarking technique, is somewhat different from the traditional critically sampled dyadic image DWT. The RDWT has been proposed for signal detection and enhancement, because of its uniform sampling rate in the spatial domain and is in some respects it is a discrete approximation to the continuous wavelet transforms. In practice, the RDWT is implemented as follows: instead of down sampling the low pass signal during each filter-bank iteration as is done in the usual DWT, the filters themselves are up sampled before performing filter convolution at each scale. The redundancy of the RDWT facilitates the identification of salient features in an image, especially image edges. Specifically, the direct multiplication of the RDWT coefficients at adjacent scales distinguishes important features from the background due to the fact that wavelet-coefficient magnitudes are correlated across scales. The coefficient-magnitude correlation is well known to exist in the usual critically sampled DWT also; however, the changing temporal sampling rate makes the calculation of an explicit correlation mask across scales much more difficult for the critically sampled DWT. The RDWT wavelet frame leads to an over complete decomposition of the signal

$$
\begin{aligned}
& d^{D W F}(k)=\left\langle g_{j}(l-k), x(l)\right\rangle l_{2} \\
& C^{D W F} d_{0}=\left\langle h_{d}(l-k), x(l)\right\rangle l_{2}
\end{aligned}
$$

where $g_{j}$ and $h_{d}$ are the analysis filter bank and $l_{2}$ is the frame of the Hilbert space. The Reconstruction formula can be mathematically expressed as follows:

$$
x(l)=\sum_{k \in z} C_{d_{0}}(k) h_{d_{0}}(l-k)+\sum_{j=1}^{d_{0}} d_{j}(k) g_{j}(l-k)
$$




\section{$3 \quad$ RDWT Watermarking Architecture}

The image watermarking can be viewed as the converse process of image denoising-in image watermarking, the watermark, which can be considered as "noise," is added to the original image. The objective is that the added "noise" be imperceptible to the human visual system (HVS) and difficult to remove by intentional or non-intentional image impairments. The RDWT aids this watermarking process by providing a mask to guide where the watermark is added. In the present technique, watermark information is embedded into the coefficients of LL and HH sub-bands of RDWT decomposition of the cover image as it provides the maximum spectrum spreading supported by cross correlation result available in Fig.1. For each watermark bit, one PN sequence of length equal to the size of LL or HH sub band is generated. If PN code is used for data embedding in LL sub band, the orthogonal code $\overline{P N}$ obtained by complementing the bits of PN code are used for data embedding in HH sub band. The use of PN and $\overline{P N}$ indicates low correlation of code patterns with the corresponding image blocks. Hadamard basis is used to decrease cross correlation among PN codes [4]. In the present technique, let $B$ denotes the binary valued watermark bit string as a sequence of $N$ bits.

$$
B=\left\{b_{1}, b_{2}, b_{3}, \ldots, b_{N}\right\}, \quad b_{i} \in(1,-1)
$$

If $I$ denotes the image block of length $M$ i.e. image transformation coefficients of length $M$, a binary valued code pattern of length $M$ is used to spread each watermark bit. Thus a set $P$ of $N$ code patterns, each of length $M$, are generated to form watermark sequence $W$ by performing the following operation:

$$
\begin{gathered}
{\left[W_{M}\right]=\sum_{j=1}^{N} b_{j}\left[P_{M}\right] j} \\
\bar{P}_{M}=\left\{\left(P_{M}\right)_{1},\left(P_{M}\right)_{2}, \ldots,\left(P_{M}\right)_{N}\right\} \\
\left(P_{M}\right)=\left\{p_{1}, p_{2}, \ldots, p_{M}\right\}
\end{gathered}
$$

where $\bar{P}_{M}$ is the set of PN sequences and $p_{1}, p_{2}, p_{3}, \ldots, p_{M}$ are elements of a PN sequence $\left(P_{M}\right)_{i}$.

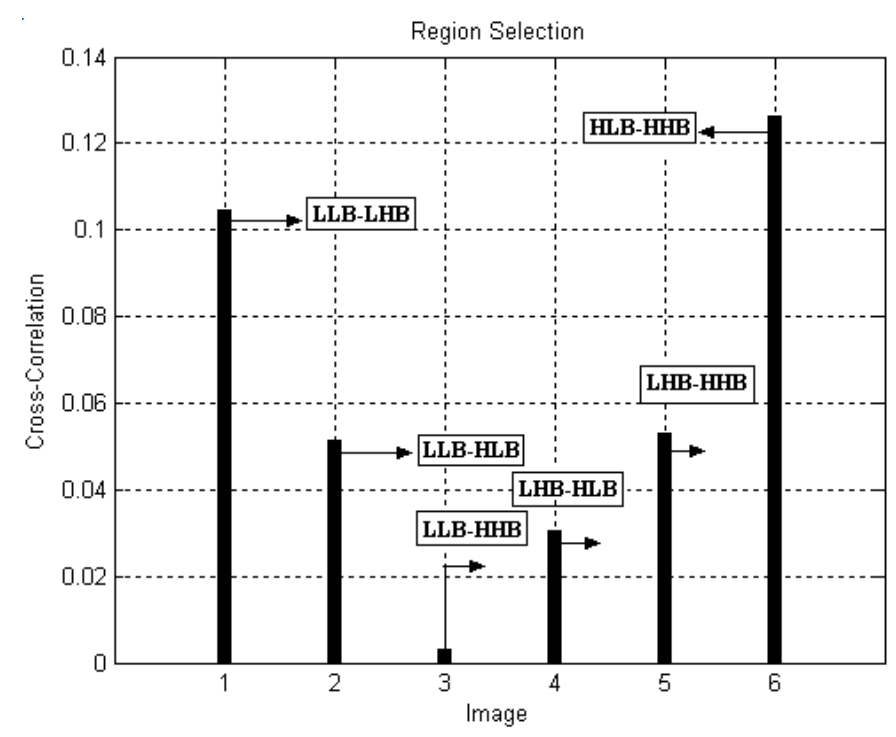

Fig. 1: Cross Correlation Among Different RDWT Sub Bands.

The watermarked image $I_{W}$ can be obtained by embedding watermark information $W$ into the image block $I$. The data embedding can be expressed mathematically as follows:

$$
\left[\left(C_{W}\right)_{M}\right]=\left[\left(C_{I}\right)_{M}\right]+\alpha\left[W_{M}\right]
$$




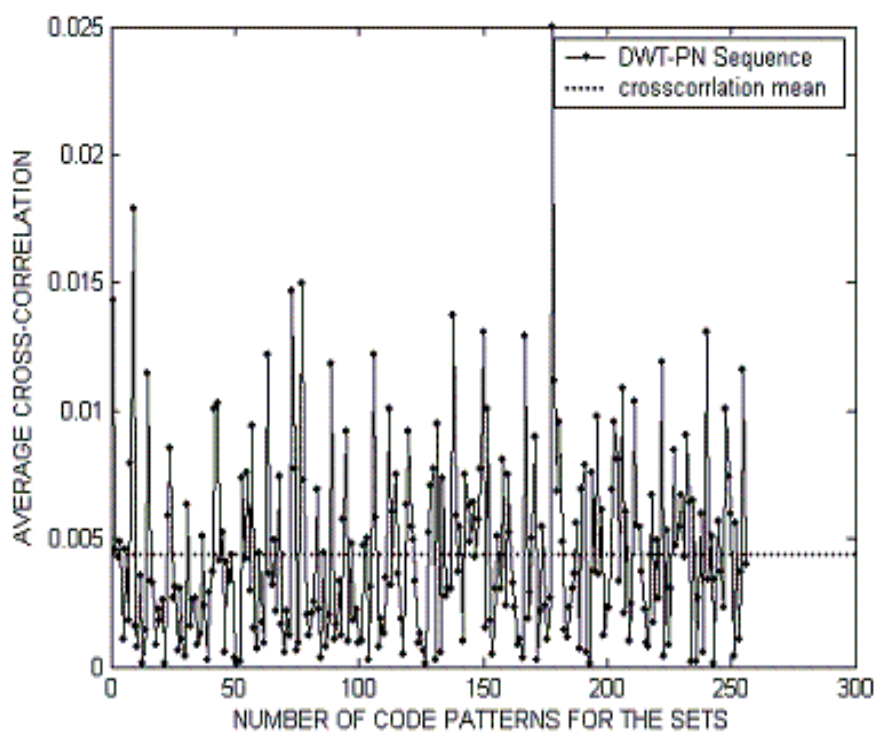

Fig. 2: Cross Correlation Between DWT Sub Band and Set of PN Sequences.

where $\alpha$ is the gain factor or modulation index and its proper choice will optimize the maximum amount of allowed distortion and minimum watermark energy needed for a reliable detection. $\alpha$ may or may not be a function of image coefficients. Accordingly SS watermarking schemes can be called as signal adaptive or non-adaptive SS watermarking. In spread spectrum watermarking, the image signal is transformed using a wavelet expansion basis,

$$
I_{I}=\sum_{i} C_{I_{i}} \psi_{i}
$$

and the watermark sequence is added to the coefficients in the wavelet transform domain to form the watermarked image signal,

$$
\begin{aligned}
& I_{W}=\sum_{i} C_{W_{i}} \cdot \psi_{i} \\
& =\sum_{i}\left(C_{I_{i}}+\alpha \cdot W_{M_{i}}\right) \cdot \psi_{i} \\
& =\sum_{i}\left(C_{I_{i}}+\alpha \cdot b_{j} P_{M j_{i}}\right) \cdot \psi_{i}
\end{aligned}
$$

In SS watermarking the detection reliability for the binary valued watermark data depends on the decision variable $\rho_{t h}$ obtained by evaluating the normalized zero-lag spatial cross covariance function between the image $I_{W}$ and each code pattern $P_{M i}$. The estimated binary bit $\hat{b}_{j}$ can be mathematically represented as follows:

$$
\widehat{b}_{j}=\operatorname{sign}\left(\frac{1}{M \alpha}\left\langle\left(C_{W}^{\prime}\right)_{M}-m_{1}\left(C_{W}^{\prime}\right)_{M}, P_{M i}-m_{1}\left(P_{M i}\right)\right\rangle(0)\right)
$$


where $m_{1}(S)$ represents the average of the sequence $S$. The symbol (0) in equation (10) indicates the normalized zero-lag cross correlation.

If $s_{k}$ represents the elements of $S$ with $k=1,2,3, \ldots, M$, then $m_{1}(S)$ can be mathematically expressed as follows:

$$
m_{1}(S)=\frac{1}{M} \sum_{k=1}^{M} s_{k}
$$

For two sequences $\mathrm{S}$ and $\mathrm{R}$, the zero lag cross correlation is given by

$$
\langle S, R\rangle(0)=\frac{1}{M} \sum_{k=1}^{M} s_{k} r_{k}
$$

where $s_{k}$ and $r_{k}$ are the elements of sequences $S$ and $R$ respectively with $k_{i}=1,2,3, \ldots, M$.

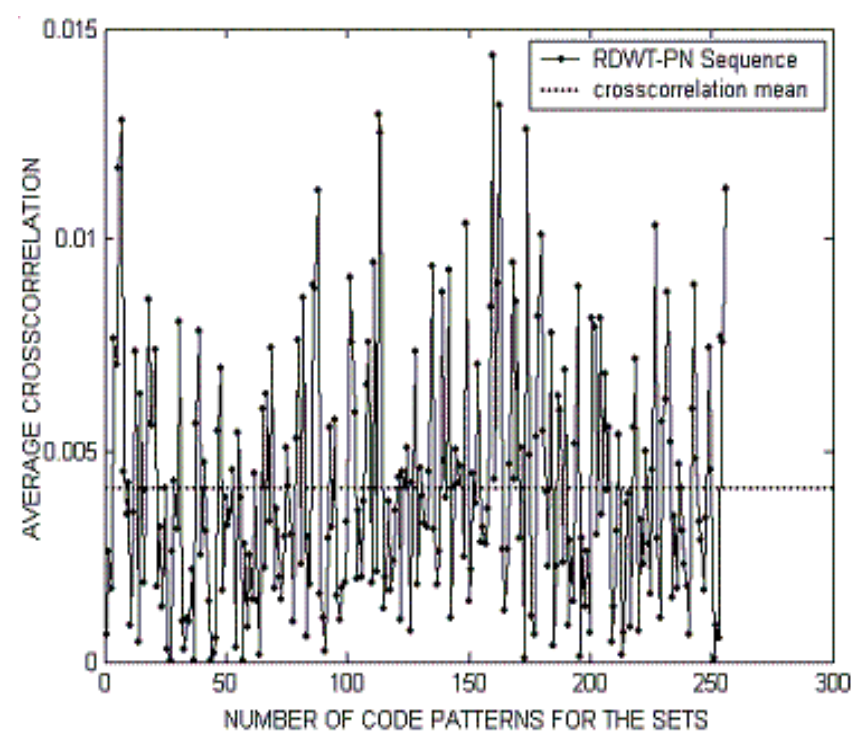

Fig. 3: Cross Correlation between RDWT Sub Band and Set of PN Sequences.

The bit $b_{i}$ is detected as ' 0 ' if $\rho_{\mathrm{th}}>0$ and as ' 1 ' otherwise. If the code patterns $P_{M i}$ are chosen so that $m_{1}\left(P_{M i}\right)=0$ for all $i$, the computation of $\rho_{\text {th }}$ becomes:

$$
\begin{aligned}
& \rho_{\text {th }}=\operatorname{sign}\left(\frac{1}{M \alpha}\left\langle\left(C_{W}^{\prime}\right)_{M}, P_{M i}\right\rangle\right) \\
& \rho_{\text {th }}=\operatorname{sign}\left(\frac{1}{M \alpha}\left\langle\left(C_{W}+d\right)_{M}, P_{M i}\right\rangle\right)
\end{aligned}
$$

where $d$ is the additive image signal impairment. Hence, without signed normalization, the covariance is:

$$
\begin{aligned}
& \rho_{\text {th }}^{\prime}=\left\langle\left(C_{W}+d\right)_{M}, P_{M i}\right\rangle \\
& =\sum_{k=1}^{A M}\left(\left(C_{W}+d\right)_{M}\right)_{k}\left(P_{M_{i}}\right)_{k}, \quad A>=1 \\
& =\sum_{k}\left\langle I_{W}^{\prime}, \psi_{k}\right\rangle\left(P_{M_{i}}\right)_{k}
\end{aligned}
$$




$$
\begin{gathered}
=\sum_{k}\left\langle\psi_{k},\left(\frac{1}{A} \sum_{l} C_{W}^{\prime} \psi_{l}\right)\right\rangle\left(P_{M i}\right)_{k} \\
=\sum_{k}\left\langle\psi_{k},\left(\frac{1}{A} \sum_{l}\left(C_{l i}+\alpha b_{j} P_{M j}+d_{1}\right) \psi_{l}\right)\right\rangle\left(P_{M i}\right)_{k} \\
=\frac{1}{A} \sum_{k} \sum_{l}\left(C_{I_{I}}+\alpha b_{j} P_{M j}+d_{l}\right)\left(P_{M i}\right)_{k}\left\langle\psi_{k}, \psi_{l}\right\rangle \\
=\frac{1}{A} \sum_{k} \sum_{l} C_{I_{l}}\left(P_{M_{i}}\right)_{k}\left\langle\psi_{k}, \psi_{l}\right\rangle+ \\
+\frac{1}{A} \sum_{k} \sum_{l} d_{l}\left(P_{M i}\right)_{k}\left\langle\psi_{k}, \psi_{l}\right\rangle+\frac{1}{A} \sum_{k} \sum_{l} \alpha b_{j}\left(P_{M j}\right)_{l}\left(P_{M i}\right)_{k}\left\langle\psi_{k}, \psi_{l}\right\rangle
\end{gathered}
$$

The above analysis indicates that code patterns used for spread spectrum watermarking should possess some specific properties [5]. Watermark detection is improved if the following conditions are satisfied:

(1) $P_{M}(i), i=1,2, \ldots, M$, should be distinct sequences with zero average.

(2) The spatial correlations $<P_{M i}, P_{M j}>, j=i$ should be minimized. Ideally, sequences $P_{i}$ and $P_{j}$ should be orthogonal whenever $j=i$.

(3) Each $P_{M i}$ for $i=1,2, \ldots ., M$ should be uncorrelated with the image block $I$ when image prediction (for estimating the image distortion) is not used before evaluating the cross correlation.

In this case the mean and variance of the correlation are,

$$
\mu_{\rho_{t h}^{\prime}}=E\left[\rho_{t h}^{\prime}\right]=\alpha M
$$

(assuming $\Psi_{i}$ have unit norm)

and

$$
\begin{gathered}
\sigma_{\rho_{t h}^{\prime}}^{2}=E\left[\rho_{t h}^{\prime}-\mu_{\rho_{t h}^{\prime}}\right]^{2}=E\left[\rho_{t h}^{\prime 2}\right]-\mu_{\rho_{t h}^{\prime}}{ }^{2} \\
=\frac{1}{A^{2}}\left[A^{2} \sum_{l=1}^{A M}{C_{I_{l}}}^{2}+d_{l}^{2}+b_{j}^{2} \alpha^{2}\left(M^{2}+2 M\right)\right]-\alpha^{2} M^{2} \\
=\frac{1}{A^{2}}\left[A^{2} \sum_{l=1}^{A M}{C_{I_{l}}}^{2}+d_{l}{ }^{2}+\alpha^{2}\left(M^{2}+2 M\right)\right]-\alpha^{2} M^{2} \\
=\sum_{l=1}^{A M}{C_{I_{l}}}^{2}+d_{l}{ }^{2}+\alpha^{2}\left(M^{2}+2 M\right)-\alpha^{2} M^{2} \\
=A\left\|I_{I}\right\|^{2}+\|S I\|^{2}+2 \alpha^{2} M, \quad A>1
\end{gathered}
$$

where $A\left\|I_{I}\right\|^{2},\|S I\|^{2}, 2 \alpha^{2} M$ are the original image signal energy , strength of the signal impairment and embedded distortion of the watermarked image signal, respectively. Since $\mathrm{m}_{1}\left(P_{M i}\right)=0 \forall i$, therefore the property (1) and (2) are readily satisfied. The figures (2) and (3) imply that correlation between RDWT image signal coefficients and $P_{M i}$ is comparatively quite low with respect to that of DWT. Hence property (3) is better satisfied for RDWT.

\section{$4 \quad$ Results and Discussion}

We test the effect of RDWT in robustness improvement over large number of benchmark images like Fishing Boat, Lena, Pills, US air force etc. We use Peak signal to Noise Ratio (PSNR) and mean Structural Similarity index (MSSIM) as representative objective measures of data imperceptibility where as relative 
entropy distance as measure of security $(\varepsilon)$. Higher PSNR values indicate better imperceptibility, although the measure does not always reflect the exact visual quality.

Now, imperceptibility is better with the higher value of MSSIM (maximum value is 1 ) where as lower the $(\varepsilon)$ value (minimum value is 0 ), better is the data security. Relative entropy (Kulback Leibler distance) distance between the cover and the watermarked image is used here as security measure of the embedded data [7]. We treat digital watermarking as a problem of digital communication and Normalized Cross Correlation (NCC) is considered as a subjective measure to quantify the robustness efficiency [1]. However, the subjective measurement depends on various factors such as the expertise of the viewers, the experimental conditions, etc. and an objective measure becomes essential to quantify the fidelity of the extracted message. One popular measure for quality assessment of the decoded data is the normalized cross correlation (NCC). For the experimental results described in this section, our RDWT watermarking technique employs a twoscale, separable 2D RDWT using length-4 min and max type wavelet filters. The parameter $\alpha$ that controls the strength of the added watermark is selected so as to achieve a desirable PSNR. We compare our RDWT technique with another transform domain technique intended to be representative of the typical approach based on the usual critically sampled DWT. In both techniques, the watermark strength is adjusted as needed to obtain a given PSNR (for these experiments, approximately 42dB). Various non malicious as well as deliberate image degradations in the form of linear, non linear filtering, image sharpening, dynamic range change, image rescaling, histogram equalization, cropping, collusion, additive white gaussian noise, lossy compression like JPEG (Fig. 4) and JPEG 2000 (Fig. 5) etc. have been simulated over the watermarked images (Fig. 6). Experimental results (Fig. 4 and 5 and Table 1, 2 and 3) show that the data embedding in RDWT sub band coefficients provides better robustness compared to DWT domain embedding although the performance of the latter is much better compared to other SS watermarking schemes implemented by using DCT and Walsh-Hadamard transformation. This can also be supported by the following mathematical expressions as follows:

Let the watermark strength $\alpha$ is adjusted in such a way that an MSE of $D$ is obtained. Then, we have

For DWT,

$$
{\sigma_{\rho_{t h}^{\prime}}}^{2}=\left\|I_{I}\right\|^{2}+\|S I\|^{2}+2 \alpha^{2} M
$$

$$
M S E_{D W T}=E\left[\left\|I_{W}-I_{I}\right\|^{2}\right]=2 \alpha^{2} M+E\left[\|S I\|^{2}\right]
$$

(Since $A=1$ for DWT)

And

$$
\begin{gathered}
\left.D=2 \alpha^{2} M+X_{S I}, \quad \text { (let } X_{S I}=E\left[\|S I\|^{2}\right]\right) \\
\alpha_{D W T}=\sqrt{\frac{D-X_{S I}}{2 M}}
\end{gathered}
$$

For RDWT,

$$
\begin{gathered}
{\sigma_{\rho_{T H}^{\prime}}{ }^{2}=A\left\|I_{I}\right\|^{2}+\|S I\|^{2}+2 \alpha^{2} M,}^{2} \quad A>1 \\
M S E_{R D W T}=E\left[\left\|I_{W}-I_{I}\right\|^{2}\right]=\frac{2 \alpha^{2} M}{A}+\frac{E\left[\|S I\|^{2}\right]}{A} \\
D=\frac{2 \alpha^{2} M}{A}+\frac{X_{S I}}{A} \\
\alpha_{R D W T}=\sqrt{\frac{A D-X_{S I}}{2 M}}
\end{gathered}
$$

Under the same signal impairment intensity and having same PSNR/MSSIM, it is quite clear from the expressions (34) and (38) that $\alpha_{\mathrm{RDWT}}$ is larger than $\alpha_{\mathrm{DWT}}$ as $A>1$ for RDWT and $A=1$ for DWT. As embedding distortion is high for RDWT than that of DWT, the detection reliability is quite better for RDWT watermarking than that of the critically sampled DWT. 


\section{Conclusion}

\begin{tabular}{|l|l|l|l|}
\hline Image & $\begin{array}{l}\text { PSNR } \\
(\mathrm{dB})\end{array}$ & SSIM & Security \\
\hline Lena & 41.565625 & 0.9795 & 0.003319 \\
\hline Fishing Boat & 42.047112 & 0.9869 & 0.003051 \\
\hline US-256 & 42.022858 & 0.9845 & 0.003127 \\
\hline Bandon & 41.137519 & 0.9734 & 0.003347 \\
\hline Cameraman & 40.612737 & 0.9712 & 0.004024 \\
\hline
\end{tabular}

Table 1: Results for PSNR, SSIM \& Security Values

\begin{tabular}{|l|l|l|l|}
\hline Attack & $\begin{array}{l}\text { PSNR } \\
(\mathrm{dB})\end{array}$ & SSIM & NCC \\
\hline $\begin{array}{l}\text { Gaussian } \\
\text { Filtering (5 }\end{array}$ times $)$ & 22.42 & 0.5373 & 0.6763 \\
\hline Sharpening & 25.98 & 0.9018 & 1 \\
\hline $\begin{array}{l}\text { Histogram } \\
\text { Equalisation }\end{array}$ & 17.15 & 0.6563 & 1 \\
\hline Rescaling & 21.46 & 0.5449 & 0.1635 \\
\hline Cropping & 21.85 & 0.8524 & 0.9755 \\
\hline Gaussian Noise & 31.59 & 0.8906 & 1 \\
\hline $\begin{array}{l}\text { Lowpass Filtering } \\
\left(3^{\text {rd }} \text { times }\right)\end{array}$ & 22.94 & 0.5811 & 0.4117 \\
\hline $\begin{array}{l}\text { Median Filtering } \\
\left(3^{\text {rd }} \text { times }\right)\end{array}$ & 25.14 & 0.7046 & 0.3925 \\
\hline $\begin{array}{l}\text { Dynamic range } \\
\text { Change }\end{array}$ & 16.55 & 0.5396 & 1 \\
\hline
\end{tabular}

Table 2: Robust ness Efficiency of Fishing Boat Image using RDWT-Max Type Wavelet Filter

\begin{tabular}{|l|l|l|l|}
\hline Attack & $\begin{array}{l}\text { PSNR } \\
(\mathrm{dB})\end{array}$ & SSIM & NCC \\
\hline $\begin{array}{l}\text { Gaussian } \\
\text { Filtering } \text { (5 }^{\text {th }} \text { times) }\end{array}$ & 22.42 & 0.5368 & 0.4763 \\
\hline Sharpening & 25.92 & 0.9045 & 0.9051 \\
\hline $\begin{array}{l}\text { Histogram } \\
\text { Equalisation }\end{array}$ & 17.15 & 0.6628 & 0.9675 \\
\hline Rescaling & 21.49 & 0.5487 & 0.0929 \\
\hline Cropping & 21.85 & 0.8521 & 0.8690 \\
\hline Gaussian Noise & 31.60 & 0.8872 & 0.8924 \\
\hline $\begin{array}{l}\text { Lowpass Filtering } \\
\text { (3 }\end{array}$ & 22.94 & 0.5799 & 0.2503 \\
\hline $\begin{array}{l}\text { Median } \text { rimes } \\
\text { times) }\end{array}$ & 25.15 & 0.7051 & 0.3433 \\
\hline $\begin{array}{l}\text { Dynamic range } \\
\text { Change }\end{array}$ & 16.52 & 0.5318 & 0.9289 \\
\hline
\end{tabular}

Table 3: Robustness Efficiency of Fishing Boat Image using RDWT-Min Type Wavelet Filter 
In this paper, we have critically analysed RDWT transform domain issue that has significant impact on imperceptibility, detection reliability and data embedding capacity in SS watermarking. It is found that data embedding in LL and $\mathrm{HH}$ sub bands (in case of RDWT) offers better resiliency against various types of image distortion than that available in DWT domain. Proposed SS watermarking scheme also offers visual and statistical invisibility and better security of the hidden data. Detection reliability is improved by increasing orthogonality among code patterns using Walsh-Hadamard basis functions. Although the reported results are based on images, the same conclusions can be extended for other kind of data like audio, music, video etc.

\section{References}

[1] I. J. Cox, J. Kilian, F. T. Leighton, and T. Shamoon, "Secure Spread spectrum watermarking for multimedia,” IEEE Transaction on Image Processing, volume 6, pp 1673-1687, 1997.

[2] J. G. Cao, J.E.Fowler, N.H.Younan, “An image-adaptive watermark based on a redundant wavelet transform”, Proceedings of the IEEE International Conference on Image Processing, Thessaloniki, Greece, October 2001, pp. 277-280.

[3] James E. Fowler, “The Redundant Discrete Wavelet Transform and Additive Noise,” IEEE Signal Processing Letters, vol. 12, pp. 629-632, September 2005.

[4] H. S. Malvar and A. F. Florencio., "Improved spread spectrum: a new modulation technique for robust watermarking,” IEEE Transaction on Signal Processing, volume 51, pp 898-905, April 2003.

[5] J. Mayer, A. V. Silverio and J. C. M. Bermudez, "On the design of pattern sequences for spread spectrum image watermarking,” International Telecommunications Symposium, Natal, Brazil.

[6] Z. Wang, A. C. Bovik, H. R. Sheikh, and E. P. Simoncelli, “ Image quality assessment: From error measurement to structural similarity,” IEEE Transaction on Image Processing, Volume 13, pp 1-14, January 2004.

[7] C. Cachin, "An information theoretic model for steganography," Proceedings of 2nd Workshop on Information Hiding, D. Aucsmith (Eds.), 1525, LNCS, Springer-Verlag, NY, May 1998.

[8] T. S. Das, A. K. Sau, and S. K. Sarkar, "Spread Spectrum Image Watermarking for Secured Multimedia Data Communication,” on International Journal of Signal Processing, Vol.3, No.3, ISSN 1304-4478, pp.148-157, (May, 2006)

[9] T. S. Das, V. H. Mankar, S. K. Sarkar, "Spread Spectrum based Robust Image Watermark Authentication," International Conference on Advanced Computing \& Communications ICACC2007, February 9-10, 2007.

[10] C. S. Burrus, R. A. Gopinath, H. Guo, "Introduction to Wavelets and Wavelet Transform," A Primer, Prentice Hall, NJ, 1997.

[11] S. P. Maity, M. K. Kundu, T. S. Das, "Robust SS watermarking with improved capacity," ScienceDirect,Elsevier, Pattern Recognition Letters 28 pp 350-356, 2007

[12] R. L. Peterson, R. E. Zeimer, D. E. Borth, “Introduction to Spread Spectrum Communications,” $P H$, Englewood Cliffs, NJ, 1995.

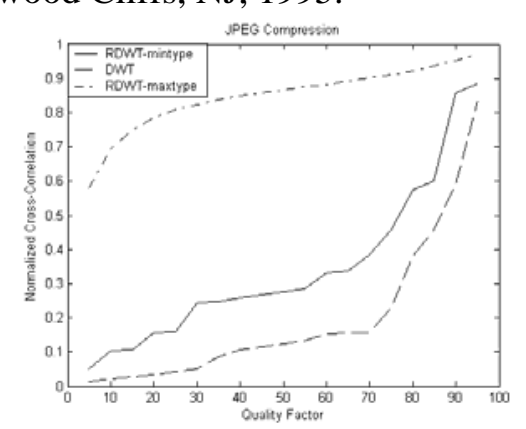

Fig. 4: Measure of Robustness against JPEG Compression.

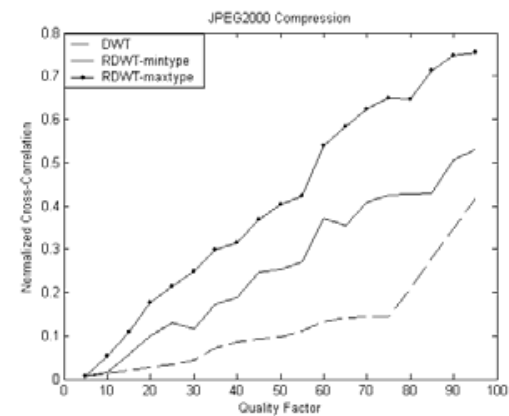

Fig. 5: Measure of Robustness against JPEG2000 Compression. 


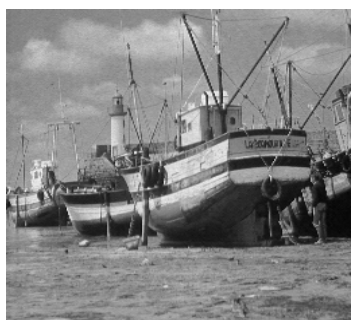

Fig. 6.1: Fishing Boat

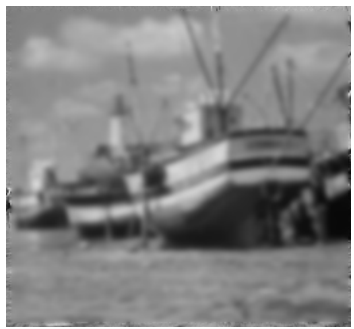

Fig.6.3: Gaussian Filtering $\left(5^{\text {th }}\right.$ times)

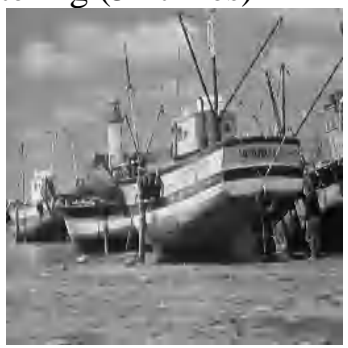

Fig. 6.5: JPEG2000

(QF: 35)

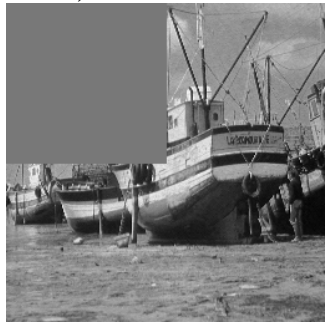

Fig. 6.7: Cropping

$(\mathrm{r}=1: 128, \mathrm{c}=1: 128)$

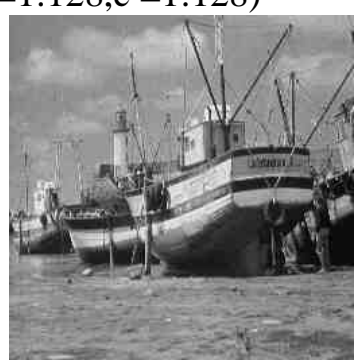

Fig.6.9: JPEG

(QF: 35)

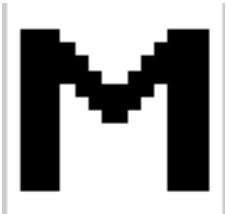

Fig. 6.2: Original Watermark

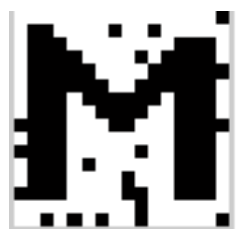

Fig. 6.4: Gaussianrecovered Watermark

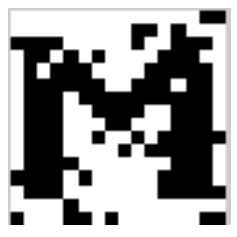

Fig. 6.6: JPEG2000recovered Watermark

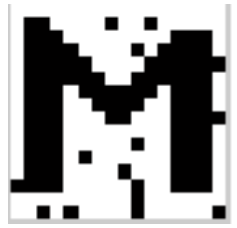

Fig. 6.8: Crop- recovered Watermark

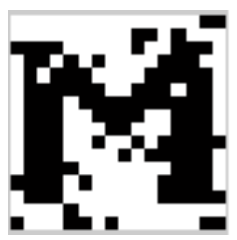

Fig. 6.10: JPEG-

recovered Watermark

Fig. 6: Robustness Efficiency under Various Impairements 